Chinese Journal of Organic Chemistry

NOTE

\title{
海南暗罗根中一个新的克罗烷型二萜
}

\author{
李小宝宋小平*陈光英*韩长日 \\ 陈文豪郑彩娟宋金明 \\ (海南师范大学化学与化工学院 热带药用植物化学教育部重点实验室 海口 571158)
}

\begin{abstract}
摘要 采用硅胶柱层析, 制备薄层层析等色谱方法, 从海南暗罗根中分离得到 8 个二萜类化合物, 分别鉴定为 polyalimide A (1), kolavenic acid (2), 2-oxo-kolavenic acid (3), 2-oxo-14,15-bisnor-3,11E-kolavadien-13-one (4), 3,4-dihydroxyclerodan-13E-en-15-oic acid (5), longimide B (6), 16-oxocleroda-3,13E-dien-15-oic acid (7), solidagonal acid methyl ester (8). 其中化合物 $\mathbf{1}$ 为新化合物，化合物 $\mathbf{5}$ 首次从番荔枝科分离得到，所有化合物首次从该植物中分离得到. 化合物 2 和 3 对人肿瘤细胞 SPCA-1, SGC-7901 和 K-562 显示微弱的细胞毒性.

关键词 暗罗属; 海南暗罗; polyalmide A
\end{abstract}

\section{A New Clerodane Diterpenoid from the Roots of Polyalthia laui Merr.}

\author{
Li, Xiaobao Song, Xiaoping* Chen, Guangying* Han, Changri
}

Chen, Wenhao Zheng, Caijuan Song, Xinming

(Key Laboratory of Tropical Medicinal Plant Chemistry of Ministry of Education, College of Chemistry and Chemical

Engineering, Hainan Normal University, Haikou 571158)

\begin{abstract}
Eight clerodane-type compounds were isolated from the roots of Polyalthia laui and purified by silica gel column chromatorgraphy and preparative thin layer chromatorgraphy (TLC). Their structures were elucidated as polyalimide A (1), kolavenic acid (2), 2-oxo-kolavenic acid (3), 2-oxo-14,15-bisnor-3,11E-kolavadien-13-one (4), 3,4-dihydroxyclerodan-13Een-15-oic acid (5), longimide B (6), 16-oxocleroda-3,13E-dien-15-oic acid (7), and solidagonal acid methyl ester (8). Compound $\mathbf{1}$ was a novel compound and compound 5 was isolated from this family for the first time, and all compounds were isolated for the first time from this plant material. Compounds $\mathbf{2}$ and $\mathbf{3}$ showed weakly inhibitory activities against SPC-A-1, SGC-7901 and K-562 human tumor cells.
\end{abstract}

Keywords Polyalthia; Polyalthia laui Merr.; polyalimide A

海南暗罗 (Polyalthia laui Merr.) 为番荔枝科 (Annonaceae) 暗罗属(Polyalthia) 植物, 为海南特有种. 在印度民间，暗罗属植物被用作传统退烧药、隍胎药、 治疗高血压等药物 ${ }^{[1]}$. 在海南民间, 该属植物被用于治 疗气滞腹痛、痛经、梅核气等疾病. 国内外学者从暗罗 属植物中得到生物碱类、二萜类、三萜类等多种化学成 分 $^{[1]}$. 本课题组对海南暗罗根进行了抗肿瘤活性篎选, 结果发现其乙醇提取物对人肿瘤细胞 SPCA-1, SGC7901 和 $\mathrm{K} 562$ 具有良好的抑制效果(SPCA-1 IC 50 值为 $44.3 \mu \mathrm{g} / \mathrm{mL}, \mathrm{SGC}-7901 \mathrm{IC}_{50}$ 值为 $6.74 \mu \mathrm{g} / \mathrm{mL}, \mathrm{K} 562 \mathrm{IC}_{50}$ 值为 $94.7 \mu \mathrm{g} / \mathrm{mL})^{[2]}$. 为深入探讨海南暗罗根的生物活性
成分, 本研究对海南暗罗根的化学成分进行了系统研 究, 从中分离得到 8 个二萜类化合物: polyalimide A (1), kolavenic acid (2), 2-oxo-kolavenic acid (3), 2-oxo-14,15bisnor-3,11E-kolavadien-13-one (4), 3,4-dihydroxy-clerodan-13E-en-15-oic acid (5), longimide B (6), 16-oxocleroda-3,13E-dien-15-oic acid (7), solidagonal acid methyl ester (8). 其中化合物 $\mathbf{1}$ 为新化合物，化合物 $\mathbf{5}$ 首次从番 荔枝科分离得到，所有化合物首次从该植物中分离得 到. 化合物 2, 3 对肿瘤细胞 SPCA-1, SGC-7901 和 K-562 具有微弱的抑制作用.

\footnotetext{
*E-mail: sxp628@126.com

Received May 15, 2013; revised May 28, 2013; published online June 4, 2013.

Project supported by the National Natural Science Foundation of China (Nos. 81160391, 21166009 and 21162009), the 973 Research Program of the Ministry of Science and Technology of China (No. 2011CB512010).

国家自然科学基金(Nos. 81160391, 21166009, 21162009)和科技部 973 计划前期研究专项(No. 2011CB512010)资助项目.
} 


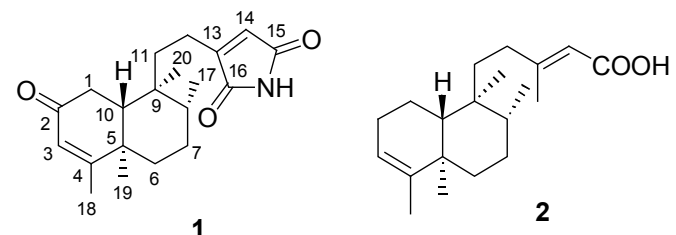<smiles>CC1=CC(=O)C[C@@]23CCC[C@]12CC/C(=C\C(=O)O)CC3</smiles><smiles>CC(=O)/C=C\C1CCCC2C(C)=CC(=O)CC12</smiles>

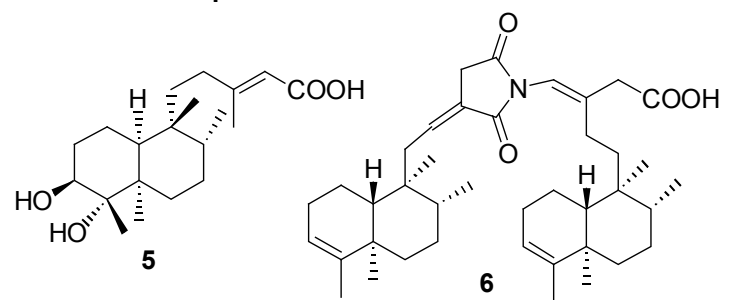

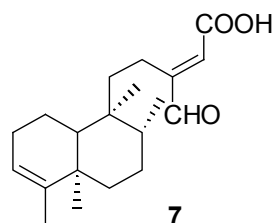

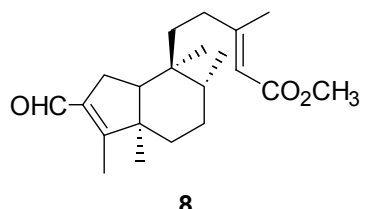

图 1 化合物 $1 \sim 8$ 的结构

Figure 1 Structures of compounds $\mathbf{1} \sim \mathbf{8}$

\section{1 结果与讨论}

\section{1 结构鉴定}

化合物 1 为白色粉末状固体, 易溶于氯仿, $254 \mathrm{~nm}$ 紫外灯下有暗斑, 与浓硫酸显黑色, $[\alpha]_{\mathrm{D}}^{25}-25.4$ ( $c$ $\left.0.036, \mathrm{CHCl}_{3}\right)$. IR 在 1631 和 $1650 \mathrm{~cm}^{-1}$ 处显示羰基和烯 键的吸收信号, $3400 \mathrm{~cm}^{-1}$ 处显示 $\mathrm{NH}$ 信号.

HR-ESIMS [calcd for $\mathrm{C}_{20} \mathrm{H}_{27} \mathrm{NO}_{3}$ 329.20603, found 329.20609]推出该化合物的分子式为 $\mathrm{C}_{20} \mathrm{H}_{27} \mathrm{NO}_{3}$, 不饱 和度为 $8 .{ }^{1} \mathrm{H}$ NMR 谱在高场区给出 4 个甲基信号 $\left[\delta_{\mathrm{H}} 1.86\right.$ $(\mathrm{s}, 3 \mathrm{H}), 1.06(\mathrm{~s}, 3 \mathrm{H}), 0.82(\mathrm{~d}, 3 \mathrm{H})$ 和 $0.77(\mathrm{~s}, 3 \mathrm{H})] ; 2$ 个次甲 基信号 $\left[\delta_{\mathrm{H}} 1.83(\mathrm{dd}, J=10.8,4.8 \mathrm{~Hz}, 1 \mathrm{H}, \mathrm{H}-10)\right.$ 和 $1.47(\mathrm{~m}$, $1 \mathrm{H}, \mathrm{H}-8)]$, 在低场区给出 1 个活泼氢信号 $\left[\delta_{\mathrm{H}} 10.72\right.$ $(\mathrm{NH})]$ 和 2 个烯氢信号 $\left[\delta_{\mathrm{H}} 6.50(\mathrm{~s}, 1 \mathrm{H})\right.$ 和 $\left.5.62(\mathrm{~s}, 1 \mathrm{H})\right]$, 另 外还给出 10 个质子信号. 在 ${ }^{13} \mathrm{C} \mathrm{NMR}$ 谱中, 共给出 20 个碳信号, 其中包括 3 个羰基碳信号 $\left(\delta_{\mathrm{C}} 198.7\right.$ 为酮羰基 碳信号, $\delta_{\mathrm{C}} 172.4$ 和 170.2 为 2 个酰胺碳信号), 2 个双键 碳信号 $\left[\delta_{\mathrm{C}} 173.0(\mathrm{C})\right.$ 和 $124.8(\mathrm{CH}) ; 150.4(\mathrm{C})$ 和 127.1 $(\mathrm{CH})], 4$ 个甲基碳信号 $\left[\delta_{\mathrm{C}} 18.6\right.$ (C-18), 18.0 (C-19), 17.4 $(\mathrm{C}-20)$ 和 15.6 (C-17)] 另外还包括 9 个碳信号 $\left(\delta_{\mathrm{C}} 45.1\right.$, $39.6,38.4,35.4,34.8,34.4,26.5$ 和 18.2). 根据以上 ${ }^{1} \mathrm{H}$ NMR 谱和 ${ }^{13} \mathrm{C} N M R$ 谱数据(表 1), 推测化合物 1 为克罗 烷型二萜类化合物, 且侧链连接在 C-9 位. 化合物 1 侧 链的结构是通过 ${ }^{1} \mathrm{H}-{ }^{1} \mathrm{H}$ COSY, HSQC 和 HMBC 确定的. 在 ${ }^{1} \mathrm{H}-{ }^{1} \mathrm{HCOSY}$ 谱中(图 2) H-11 和 H-12 有相关信号. 在 $\mathrm{HMBC}$ 谱中(图 2), H-14 与 C-15, C-16 和 C-12 相关确定 了顺丁烯二酰亚胺部分的结构, 且此部分结构与 C-12 相连. 该化合物的相对构型是通过耦合常数和 NOESY 谱确定. 在 ${ }^{1} \mathrm{H}$ NMR 谱中, $J_{10-1 \mathrm{ax}}=10.8 \mathrm{~Hz}$ 和 $J_{10-1 \mathrm{eq}}=4.8$ $\mathrm{Hz}$ 确定 $\mathrm{AB}$ 环为反式连接. 化合物 $\mathbf{1}$ 的相对构型是通过 与该物种中分离获得的已知克罗烷型二萜类化合物的 ${ }^{1} \mathrm{H}$ NMR 谱和 ${ }^{13} \mathrm{C}$ NMR 谱数据比较以及 NOESY 谱确定
表 1 化合物 1 的核磁共振谱图数据(400/100 MHz, DMSO- $\left.d_{6}\right)$ Table 1 NMR spectroscopic data $\left(400 / 100 \mathrm{MHz}, \mathrm{DMSO}-d_{6}\right)$ for compound 1

\begin{tabular}{cll}
\hline Position & ${ }^{1} \mathrm{H}$ NMR $(J$ in $\mathrm{Hz})$ & ${ }^{13} \mathrm{C} \mathrm{NMR}$ \\
\hline 1 & $2.22, \mathrm{dd}(16.8,4.8)$ & $34.7, \mathrm{CH}_{2}$ \\
& $2.40, \mathrm{dd}(16.8,10.8)$ & \\
2 & & $198.7, \mathrm{C}=\mathrm{O}$ \\
3 & $5.62, \mathrm{~s}$ & $124.8, \mathrm{CH}$ \\
4 & & $173.0, \mathrm{C}$ \\
5 & & $39.6, \mathrm{C}$ \\
6 & $1.73 \sim 1.76, \mathrm{~m}$ & $34.5, \mathrm{CH}_{2}$ \\
7 & $1.44 \sim 1.50, \mathrm{~m}$ & $26.5, \mathrm{CH}_{2}$ \\
8 & $1.44 \sim 1.50, \mathrm{~m}$ & $35.4, \mathrm{CH}$ \\
9 & & $38.4, \mathrm{C}$ \\
10 & $1.83, \mathrm{dd}(10.8,4.8)$ & $45.1, \mathrm{CH}$ \\
11 & $1.44 \sim 1.50, \mathrm{~m}$ & $34.5, \mathrm{CH}_{2}$ \\
12 & $1.99 \sim 2.04, \mathrm{~m}$ & $18.2, \mathrm{CH}_{2}$ \\
13 & & $150.4, \mathrm{C}$ \\
14 & $6.50, \mathrm{~s}$ & $127.1, \mathrm{CH}$ \\
15 & & $172.4, \mathrm{C}=\mathrm{O}$ \\
16 & & $170.2, \mathrm{C}=\mathrm{O}$ \\
17 & $0.82, \mathrm{~d}(8.8)$ & $15.6, \mathrm{CH}_{3}$ \\
18 & $1.86, \mathrm{~s}$ & $18.6, \mathrm{CH}_{3}$ \\
19 & $1.06, \mathrm{~s}$ & $18.0, \mathrm{CH}_{3}$ \\
20 & $0.77, \mathrm{~s}$ & $17.4, \mathrm{CH}_{3}$ \\
$\mathrm{NH}$ & $10.72, \mathrm{~s}$ & \\
\hline & &
\end{tabular}

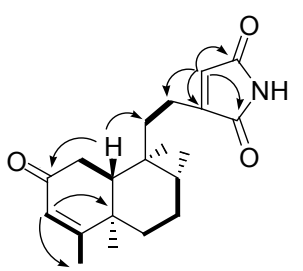

图 2 化合物 1 的 ${ }^{1} \mathrm{H}-{ }^{1} \mathrm{H} \operatorname{COSY}(-)$ 相关和主要的 $\mathrm{HMBC}$ $(\rightarrow)$ 相关

Figure $2{ }^{1} \mathrm{H}-{ }^{1} \mathrm{H} \operatorname{COSY}(\longrightarrow)$ and key $\operatorname{HMBC}(\rightarrow)$ correlations for compound 1

的. 在 NOESY 谱中(图 3), H-17 与 H-19 和 H-20 有相关, 


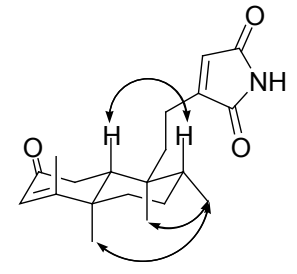

图 3 化合物 $\mathbf{1}$ 的 NOESY 相关

Figure 3 NOESY correlations of 1

与 $\mathrm{H}-10$ 和 $\mathrm{H}-8$ 没有相关信号, 说明 H-17, H-19 和 H-20 在 $\alpha$ 位, H-10 与 H-8 在 $\beta$ 位. 至此确定化合物 $\mathbf{1}$ 的结构 为 polyalimide $\mathrm{A}$.

已知化合物 kolavenic acid (2) ${ }^{[3]}$, 2-oxo-kolavenic acid $(3)^{[4]}$, 2-oxo-14,15-bisnor-3,11E-kolavadien-13-one $(4)^{[5]}, 3$,4-dihydroxyclerodan-13E-en-15-oic acid $(\mathbf{5})^{[6]}$, longimide B $(6)^{[7]}, 16$-oxocleroda-3,13E-dien-15-oic acid $(7)^{[8]}$, solidagonal acid methyl ester $(\mathbf{8})^{[9,10]}$ 的结构是通过 与文献数据比较确定的.

\section{2 抗肿瘤活性测试}

MTT 法评价化合物 1 4 对肿瘤细胞增殖抑制作用. 将对数生长期的 SPCA-1(人肺癌细胞)、SGC-7901(人胃 癌细胞)和 K-562(人白血病细胞)配制细胞悬液, 分别接 种于 96 孔培养板; 细胞贴壁后分别加入用 PBS 配制的 浓度梯度的 $\left(0.1,1,5,10,50,100 \mu \mathrm{mol} \cdot \mathrm{L}^{-1}\right.$ 六个浓度 $)$ $20 \mu \mathrm{L}$ 化合物 1 4. 每组设 5 个复孔，同时设空白对照 和溶媒(DMSO)对照, 置于 $37.0{ }^{\circ} \mathrm{C} 、 5 \% \mathrm{CO}_{2}$ 、饱和湿度 条件下, 培养 $44 \mathrm{~h}$ 后, 每孔加入 $50 \mu \mathrm{L}$ MTT 溶液(1 $\mathrm{mg} / \mathrm{mL}$, PBS 配制), 作用 $4 \mathrm{~h}$ 后弃上清, 每孔加入 $150 \mu \mathrm{L}$ DMSO, 震荡 $10 \mathrm{~min}$, 在酶联免疫检测仪上选择 $570 \mathrm{~nm}$ 波长处测吸光 $A$ 值, 抑制率 $(\%)=$ (对照组 $A$ 值一实验组 $A$ 值)/对照组 $A$ 值 $\times 100 \%$, 结果表明化合物 $\mathbf{2} \sim 3$ 对三种 肿瘤细胞有微弱的增殖抑制作用(表 2).

表 2 化合物 $1 \sim 4$ 对肿瘤细胞的抑制活性

Table 2 Cytotoxic activities of compounds $\mathbf{1} \sim \mathbf{4}$

\begin{tabular}{cccc}
\hline \multirow{2}{*}{ 化合物 } & \multicolumn{3}{c}{$\mathrm{IC}_{50} /\left(\mu \mathrm{g} \cdot \mathrm{mL}^{-1}\right)$} \\
\cline { 2 - 4 } & $\mathrm{SPCA}-1$ & $\mathrm{SGC}-7901$ & $\mathrm{~K}-562$ \\
\hline $\mathbf{1}$ & 94.5 & 96.76 & 62.73 \\
$\mathbf{2}$ & 15.6 & 17.2 & 18.9 \\
$\mathbf{3}$ & 49.0 & 38.0 & 26.9 \\
$\mathbf{4}$ & $>200$ & $>200$ & $>200$ \\
丝裂霉素 $\mathrm{C}^{a}$ & 1.68 & 3.61 & 16.56 \\
\hline 丝裂䨰素 $\mathrm{C}$ 为阳性对照. & \multicolumn{3}{c}{}
\end{tabular}

\section{2 结论}

运用硅胶柱色谱、凝胶柱色谱、制备薄层色谱等分 离方法, 从海南暗罗根中分离得到 8 个二萜类化合物: polyalimide A (1), kolavenic acid (2), 2-oxo-kolavenic acid
(3), 2-oxo-14,15-bisnor-3,11E-kolavadien-13-one (4), 3,4dihydroxyclerodan-13E-en-15-oic acid (5), longimide B (6), 16-oxocleroda-3,13E-dien-15-oic acid (7), solidagonal acid methyl ester (8). 其中化合物 $\mathbf{1}$ 为新化合物，化合物 5 首次从番荔枝科分离得到，所有化合物首次从该植物 中分离得到. 化合物 2 和 3 对人肿瘤细胞 SPCA-1, SGC-7901 和 K-562 显示微弱的细胞毒性.

\section{3 实验部分}

\section{1 仪器与试剂}

Bruker AV-400 MHz型核磁共振仪; EYELA N-1001 旋转蒸发仪; EYELA COOLACE CA-IIII 冷却水循环装 置; YOKO-ZX 紫外分析暗箱; 薄层硅胶 $\mathrm{GF}_{254}$; 柱色谱 硅胶 200 300 目; 色谱柱(各种规格普通玻璃加压柱); 色谱试剂均为分析纯. 海南暗罗药材采自海南昌江县霸 王岭自然保护区, 经海南师范大学生命科学学院钟琼芯 副教授鉴定为番荔枝科暗罗属植物 Polyalthia plagioneura. 标本现存于海南师范大学省部共建教育部热 带药用植物化学重点实验室.

\section{2 实验方法}

海南暗罗根 $15 \mathrm{~kg}$ 用 $75 \%$ 的乙醇浸泡, 加热回流两 小时, 过滤, 滤液 $60{ }^{\circ} \mathrm{C}$ 减压浓缩至棕色干浸膏 $1.2 \mathrm{~kg}$. 将浸膏混悬于水中, 依次用石油醚、氯仿、乙酸乙酯进 行萃取, 回收溶剂, 得石油醚部位 $200 \mathrm{~g}$, 氯仿部位 431 $\mathrm{g}$, 乙酸乙酯部位 $120 \mathrm{~g}$.

石油醚部位浸膏 $(200 \mathrm{~g})$, 进行硅胶柱层析, 石油 醚-乙酸乙酯梯度洗脱，合并相同流份，得到石油醚洗 脱部分(Fr.1 Fr.10), Fr.4 经硅胶柱层析, 石油醚-乙酸 乙酯 $(V: V=20: 1 \sim 0: 1)$ 洗脱得到化合物 $2(27.6 \mathrm{mg})$, $3(3.5 \mathrm{mg})$ 和 $5(5.5 \mathrm{mg})$. 氯仿部位 $(431 \mathrm{~g})$ 进行硅胶柱层 析，石油 醚一乙酸乙酯梯度洗脱，合并相同流份，得到 氯仿洗脱部分 $(F r . A \sim F r . J) . F r . E$ 用氯仿-乙酸乙酯梯度 洗脱得到三个组分, 用氯仿一乙酸乙酯 $(V: V=8: 1)$ 进 行洗脱得到化合物 $1(6.3 \mathrm{mg})$, 用氯仿一乙酸乙酯 $(V: V$ $=6: 1)$ 为展开剂进行薄层层析, 得到化合物 $6(3.3 \mathrm{mg})$ 和 $8(5.6 \mathrm{mg})$; Fr.F 用氯仿 - 甲醇 $(V: V=100: 1 \sim 15: 1)$ 洗脱得到 2 个组分, 组分 1 经制备薄层层析, 氯仿一甲醇 $(V: V=1: 10)$ 为展开剂, 得到化合物 $7(6.1 \mathrm{mg})$. 乙酸 乙酯部位 $(120 \mathrm{~g})$ 进行硅胶柱层析, 石油醚-乙酸乙酯梯 度洗脱，合并相同流份，得到氯仿洗脱部分 $(F r .1 \sim$ Fr.10), Fr.5 用氯仿一乙酸乙酯 $(V: V=10: 0 \sim 0: 1)$ 洗脱 得到化合物 4 (3.6 mg).

\section{References}

[1] Padmaa, M. P.; Khosa, R. L. J. Pharm. Res. 2009, 2(4), 594. 
[2] Yuan, Y.; Huang, G. J.; Wang, T. S.; Chen, G. Y. Phytother. Res. 2010, 27, 1.

[3] Salah, M. A.; Bedir, E. J. Agric Food Chem. 2003, 51(26), 7607.

[4] Avila, D.; Medina, J. D. Phytochemistry 1991, $30(10), 3474$.

[5] Kabir, S.; Rahman, M. S.; Sarwaruddin, A. M. Nat. Prod. Commun. 2010, 5(10), 1543.

[6] Cavin, A. L.; Hay, A. E.; Marston, A.; Evans, H. S. J. Nat. Prod 2006, 69(5), 768.
[7] Sashidhara, K. V.; Singh, S. P.; Kant, R.; Maulik, P. R.; Sarkar, J. Bioorg. Med. Lett. 2010, 20(19), 5767.

[8] Kijjoa, A.; Pinto, M. M. M.; Pinto, P. M. M.; Herz, W. Phytochemistry 1993, 34(2), 457.

[9] Bohlmann, F.; Singh, P.; Singh, R. K.; Joshi, K. C.; Jakupovic, J. Phytochemistry 1985, 24(5), 1114.

[10] Tori, M.; Katto, A.; Sono, M. Phytochemistry 1999, 52(3), 487.

(Cheng, F.) 